\title{
Biodegradable Sodium Alginate and Lignosulphonic Acid Blends: Characterization and Swelling Studies
}

\author{
S. Giridhar Reddy, Akanksha Saxena Pandit \\ Bangalore Campus, Amrita Vishwa Vidyapeetham
}

\begin{abstract}
In this article, Sodium alginate and Lignosulphonic acid blends were prepared in different weight ratios by solution casting method. Swelling experiments were conducted for Sodium alginate and its blends by adding calcium chloride for various intervals in a pH 7.4 medium. The strength of the films increases after their treatment with $\mathrm{CaCl}_{2}$ at higher intervals forming an alginate network. The SA/LS blend (80/20) is subjected for sorption studies at variable $\mathrm{pH} 1.2 \& 7.4$, in order to test the suitability of the blends for controlled drug delivery. Further, experiments were conducted for de-crosslinking using $0.1 \mathrm{M} \mathrm{HCl}$. The solvent uptake for the blends decreased with increasing LS content in the blends. Blends were investigated by X-ray diffraction and Fourier Transform Infra-Red spectroscopy in order to study the crystallinity and molecular interactions. XRD confirms the reduction in cluster space in blends leading to lower uptake of solvent.
\end{abstract}

Keywords: Sodium alginate, blends, swelling, biomaterials, water soluble polymers, crosslinking.

\section{Introduction}

Sodium alginate (SA) blends are the biodegradable polymers that are soluble in water and various other aqueous solvents. Their application in various biomedical fields has been a major area of study over the last half century. The sodium alginate hydrogels are largely used due to their high biocompatibility ${ }^{[1]}$. Over the past year, a large part of the work in the biomedical field has revolved around applications such as carriers for the modified release of drugs and bioactive macromolecules ${ }^{[1]}$. Recently, the application of alginate hydrogels as scaffolding materials has attracted much attention in the areas of tissue engineering ${ }^{[2]}$. Alginates are an interesting family of polymers that have been studied for a wide variety of biomedical applications ${ }^{[2-4]}$. This interest in the alginates is primarily due to its relative ease with which they can undergo gelation with divalent cations under the very mild conditions suitable for biomacromolecules and living cells ${ }^{[5,6]}$. But alginate hydrogels possess a limited mechanical stability under physiological conditions ${ }^{[5]}$. For biomedical applications aimed at vivo, the mechanical properties are important because they must possess sufficient mechanical strength and flexibility to withstand compressional forces from the surrounding tissues in vivo without fear of collapse. To improve the mechanical stability of membrane, Sodium alginate (SA) needs to be blended with other polymer such as Lignosulphonic acid (LS). LS is a natural biopolymer obtained as a plant byproduct formed from sulphite cooking of wood and is large tonnage wastes from pulp and paper industry. In this study, we aimed to prepare blends of sodium alginate and sodium salt of lignosulphonic acid from which blends with enhanced stability under physiological conditions can be obtained via ionically cross-linking formation in calcium chloride solution. Sorption experiments are conducted for SA and blends for water uptake. The sorption technique is a good tool for the crystalline characterization of polymer blends $s^{[7,8]}$. The analysis of the equilibrium sorption by a blend can provide information on polymer-polymer interactions and morphology of the system. Particular attention has been given to the effects of blend ratio and solvent uptake and the correlation of the results with the morphology of the matrices using XRD and FTIR characterization. Finally, SA/LS (80/20) blend is studied for water uptake in variable $\mathrm{pH} 1.2$ and $\mathrm{pH} 7.4$ for possible use in oral delivery of drugs along the Gastro intestinal tract.

\section{Experimental}

\section{Chemicals}

Sodium alginate $(\mathrm{MW}=300000 \mathrm{gm} / \mathrm{mol})$ and lignosulphonic acid $(\mathrm{MW}=50000 \mathrm{gm} / \mathrm{mol})$ were used as obtained from Sigma Aldrich Bangalore India Limited. Calcium chloride and buffer capsules of $\mathrm{pH} 1.2$ and $\mathrm{pH} 7.4$ were used as received from SD Fine Chem Ltd.

\section{Preparation of blends}

The aqueous solution of alginate (sodium alginate solution with a concentration of $1 \mathrm{wt}$. (\%) was prepared by stirring preliminary swollen sodium alginate in distilled water for 3 hours) and sodium lignosulphonic acid were mixed in various compositions $(100 / 0$, $80 / 20 \& 60 / 40$ by weight). The sample films were cast by pouring the compositions on glass substrates, dried at room temperature for 72 hours, and then dried at $60{ }^{\circ} \mathrm{C}$ to constant weight in a vacuum oven $^{[9]}$. The dried films of thickness $0.2 \mathrm{~mm}$ were obtained and the blends were cut in to circular shape of diameter $1.9 \mathrm{~cm}$ as reported in ${ }^{[10]}$.

\section{Curing SA-LS blends using calcium chloride as crosslinking agent}

The blends are cross-linked by dipping in $2 \% \mathrm{CaCl}_{2}$ solution for various intervals and then allowed to dry at $30{ }^{\circ} \mathrm{C}$ in a dust free chamber till they attained constant weight. Here it should be noted that mode of drying process affects the stability of blends. The drying of blends at higher temperature may result in surface cracking which can facilitate the surface erosion upon rehydration. This will ultimately affect the swelling/degradation behavior. Experimental conditions were maintained uniform throughout the investigations.

\section{Characterisation}

\section{Swelling studies of blends for water uptake}

The swelling behavior of the cross-linked films was measured, based on the solvent uptake by the blends in different $\mathrm{pH}$ solutions 
at the room temperature. Further, blends are wiped with tissue paper to remove water on the blends surface. The 'Weight change' was monitored at different time-intervals till the blends showed constant weight. The fractional weight change was transformed to percentage using the following empirical relationship ${ }^{[11]}$ :

Percentage of Swelling $=($ Final weight - Initial weight $) /$ Initial weight $\times 100$.

The measurements were made in triplicate and average data was used for calculations.

\section{X-Ray diffraction studies}

A Brucker D8 advanced powder X-ray diffractometer was used to study the solid state morphology of SA, LS and SA/LS Blends in powdered form. X-rays of $1.548 \AA$ wavelengths were generated by a $\mathrm{Cu} \mathrm{K} \alpha$ source. The angle of diffraction was varied from $5^{\circ}$ to $60^{\circ}$ to identify the change in the crystal structure and intermolecular distances between the intersegmental chains in blends.

\section{Fourier transform infrared spectroscopy (FT-IR) studies}

FT-IR spectra of the blends were measured on a Nicolet impact 400 FT-IR spectrophotometer. The samples were prepared by making $\mathrm{KBr}$ pellets containing 3 wt. (\%) of materials.

\section{Results and Discussions}

\section{Effect of crosslinking on blends}

The blends are cured, in order to investigate the effect of Calcium Chloride and alginate content on the swelling behavior of blends. We synthesized three blends of the proportion SA/LS blend (100/0, $80 / 20$, and 60/40). In order to investigate, water uptake behavior of SA/LS blend (80/20) samples were cross linked for 10, 20, 30 and 60 minutes was studied. The swelling studies were conducted using buffer solution ( $\mathrm{pH}$ 7.4) as penetrate. All swelling behaviors are plotted on the average of three trials. It has been reported, that maximum time required for calcium ions induced crosslinking of alginate is nearly 30 minutes. In order to investigate this aspect the SA/LS blends 80/20 were cured for, 10, 20, 30 and 60 minutes. The swelling results are depicted in Figure 1a. The Figure 1a clearly indicates that blend films exhibits different swelling/degradation behavior. The SA/LS (80/20) blend cured for 10 minutes exhibits maximum swelling nearly $430 \pm 10 \%$ in one hour and then starts to disintegrate slowly and finally dissolves in 15 hours, whereas swelling decreases at higher intervals of crosslinking ie $320 \pm 12 \%$ for 20 minutes curing and $220 \pm 10 \%$ for 30 minutes curing. The swelling decreases drastically for 60 minutes curing ie about $40 \pm 10 \%$. The above experimental data reveals one important fact that in the present system, the crosslinking processes is not

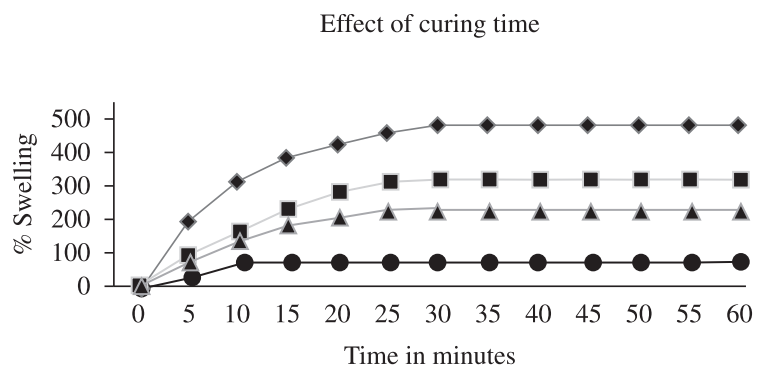

(a) completed in 30 minutes. Perhaps it requires almost 60 minutes for complete curing. It has been reported that alginate cross-linked at higher intervals provide mechanical strength to the films due to crosslinking of polyguluronate residues in alginates ${ }^{[12]}$.

\section{Effect of alginate content}

The effect of SA content on swelling behavior of the blends was studied. The results are shown in Figure 1b. The pure sodium alginate (100/0) and SA/LS blends (80/20 and 60/40), cured for 30 minutes using $2 \%$ Calcium chloride solution were swelled using buffer solution of $\mathrm{pH} 7.4$ as penetrate. The Figure $1 \mathrm{~b}$ clearly indicates, that blend films exhibit different swelling/degradation behavior. The Pure SA (100/0) exhibit maximum swelling of $500 \pm 12 \%$ of water uptake in buffer solution ( $\mathrm{pH} 7.4)$. However, the swelling decreases in SA/LS blends (80/20 \& 60/40). SA/LS blend $(80 / 20)$ exhibits moderate swelling i.e. $220 \pm 12 \%$ in one hour and then starts to disintegrate slowly after 2 hours and finally dissolves in 15 hours. On the other hand SA/LS blends (60/40) cured for same time shows a lower water uptake ie nearly $110 \pm 10 \%$ in 60 minutes and stable up to 48 hours. It has been reported that the blends has relatively more cross linked structure and hence they exhibit lower water uptake due to tight structure in the intermolecular chain; they exhibit minimum swelling ${ }^{[13]}$.

\section{Swelling behavior in $\mathrm{pH} 1.2$ and $\mathrm{pH} 7.4$}

In order to test the suitability of the blends for gastrointestinal drug delivery, the swelling behavior of SA/LS blend in ratio of 80/20, cross linked for 30 minutes using calcium chloride as crosslinking agent was studied. The SA/LS (80/20) blend was subjected for swelling for water uptake in $\mathrm{pH} 7.4$ and $\mathrm{pH}$ 1.2. From Figure 2a, it is found that swelling decreases drastically in acidic medium $100 \pm 4 \%$ in 30 minutes and then do not swell further. This is well expected because alginates do not swell significantly at low $\mathrm{pH}^{[15]}$. When a dosage is administered orally, goes to the stomach and after residing there for a definite time-period, it passes on to small intestine and then finally to colon. In this way, the polymer loaded with drug has to get exposed to a sharp $\mathrm{pH}$ change in the range 1-2 (gastric fluid) to 7-8 (intestinal fluid). Therefore, in order to mimic the transition of proposed SA/LS blend (80/20) from mouth to colon, they should be exposed to the media of varying $\mathrm{pH}$. Relying on the data given by a group of pharmaceutical researchers regarding the transit time of a dosage form along GI tract ${ }^{[14]}$, we exposed the blend to medium of $\mathrm{pH} 1.2$ for a period of 3 hours and then transferred the same blend in the phosphate buffer of $\mathrm{pH} 7.4$ for rest of the time. The results of swelling measurements have been depicted in Figure $2 b$. It is clear that the water uptake of the blend was nearly $100 \pm 4 \%$ in the acidic solution in first 30 minutes and did not swell further. When

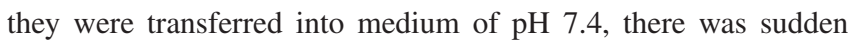

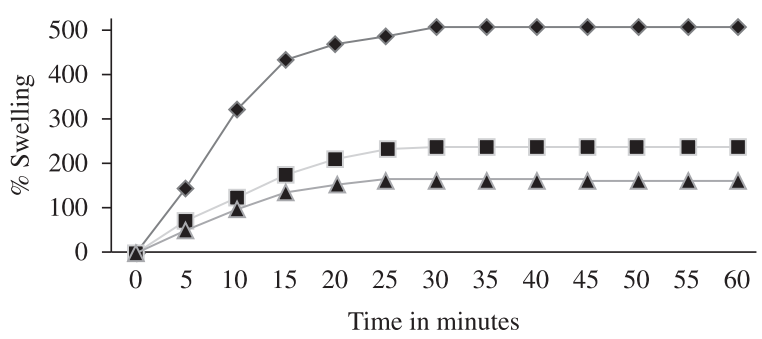

(b)

Figure 1. a) Effect of crosslinking on $\%$ swelling by NaAg/NaLS $(80 / 20)$ blend at $10(-\bullet), 20(-), 30(-\bullet)$ and $60(-\bullet)$ minutes exposure for CaCl ; b) Effect of NaAlg on \% swelling by NaAlg/NaLS blend 100/0 (- $), 80 / 20(-)$ and 60/40 ( - - ) exposure for $\mathrm{CaCl}_{2}$ for 30 minutes. 
rise in water uptake. The blend swelled to nearly $400 \pm 9 \%$ in the next 1 hour and attained equilibrium water uptake of $400 \pm 23 \%$ in a total duration of 4 hours, then started to disintegrate in next 1 hour. This can be explained as follows: when the blend is placed in the medium of $\mathrm{pH} 1.2$, they exhibit almost minimum swelling (i.e. nearly $100 \pm 4 \%$ water uptake). However, on transferring them into phosphate buffer medium of $\mathrm{pH} 7.4$, they begin to absorb water at faster rate. This is due to the fact that their stay in the medium of $\mathrm{pH} 1.2$ results in acid - catalyzed hydrolysis of alginates into low molecular alginic acid ${ }^{[16]}$. Moreover, in acidic $\mathrm{pH}$, the protonation of carboxylate groups, attached at polyguluronate blocks of alginate results in decrease in ionic crosslinking of the blend. So when this

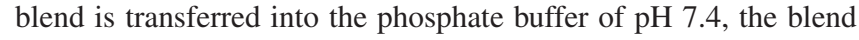
begins to take up water. The ion-exchange (although at a slower rate) between $\mathrm{Ca}^{+2}$ ions of blend and $\mathrm{Na}+$ ions of phosphate buffer also makes the structure loose, thus enhancing the uptake of water. Finally, when the blend attains maximum swelling (which is nearly $400 \pm 9 \%$ ) low molecular weight segments of alginic acid and linear chains of LS become unable to retain the hydrated structure and hence the blend begins to disintegrate. This finally causes total dissolution of the blend.

\section{Effect of treatment with $0.1 \mathrm{M} \mathrm{HCl}$}

In order to investigate the effect of acid treatment on the swelling behavior of the blends, the SA/LS blend $(80 / 20)$ is treated with $0.1 \mathrm{M} \mathrm{HCl}$ for definite time periods, i.e. for 10, 20 and 30 minutes and their swelling behavior was studied in the phosphate buffer solution of $\mathrm{pH} 7.4$ at $25{ }^{\circ} \mathrm{C}$. The results as depicted in Figure 2c reveal that the blend treated for 30 minutes with $0.1 \mathrm{M} \mathrm{HCl}$ demonstrates highest water uptake of $430 \pm 10 \%$ in 30 minutes then begin to disintegrate and finally dissolve completely, while the blend treated for 20 minutes, swell to nearly $400 \pm 10 \%$ in the same time period. The untreated blend exhibited minimum water uptake. The observed findings may be explained as below. The SA/LS (80/20) blend exposed for sufficiently longer time (i.e. 30 minutes) in $0.1 \mathrm{M} \mathrm{HCl}$, the alginate undergoes an appreciable degree of hydrolysis. The degree of crosslinking is also reduced to a greater extent. When this blend is put in solution of $\mathrm{pH} 7.4$, they begin to take up water with subsequent dissolution of alginic acid segments. This result in relatively higher water uptake $(430 \pm 10 \%$ water uptakes in 30 minutes) and then the blend begin to disintegrate and finally dissolve. The untreated blend exhibit minimum water uptake due to highly cross-linked structure and do not show any tendency to disintegrate.

\section{X-Ray diffraction studies}

X-ray diffraction studies provide information on the nature of the compound as well as on the spacing between the clusters of the polymer chains. On blending, the polymer chains are in close compaction with one another and thus a reduction in the cluster space may be encountered. The X-ray diffractograms of pure SA, LS and SA/LS blend membranes, shown in Figure 3. Figures 3a, d are diffractograms of pure SA and LS has sharp peaks, and 3b, c has no sharp peaks indicating the amorphous nature of the blend membranes $^{[13]}$. The XRD spectra of the SA/LS: 80/20 (Figure 3b) exhibits a broad peak at $2 \theta=43^{\circ}$ and $49^{\circ}$ which may be due to the intermolecular interaction between SA and LS. The SA/LS $(60 / 40)$ \& SA/LS (80/20) blend membranes appears to be more

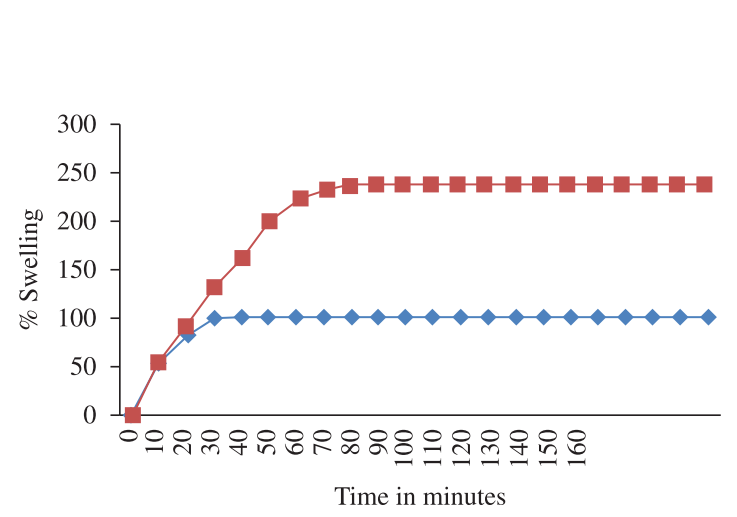

(a)

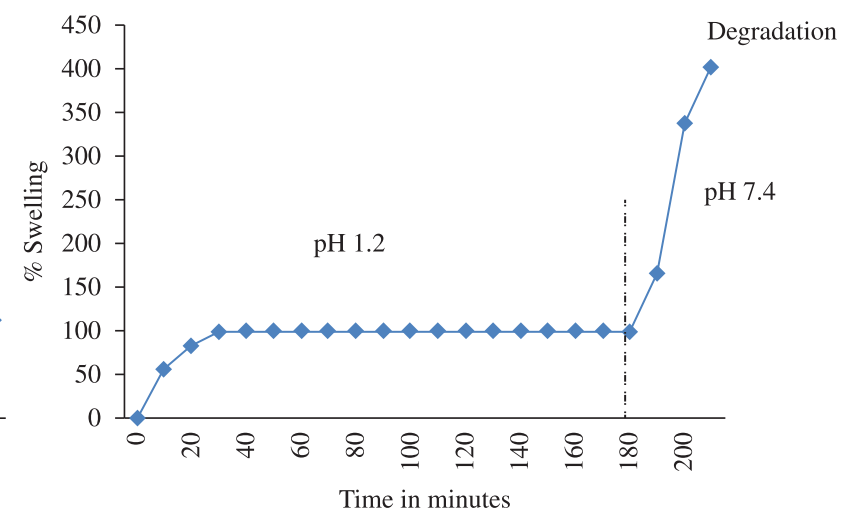

(b)

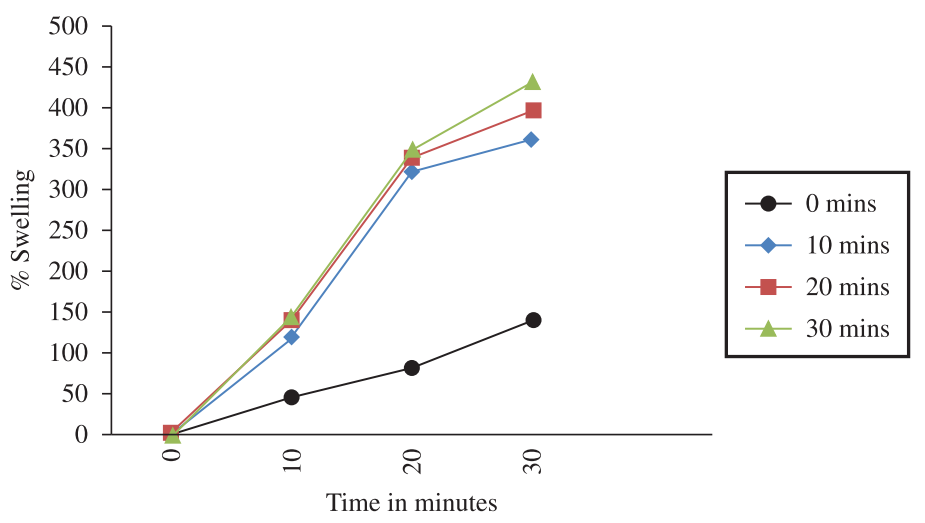

(c)

Figure 2. A composite depiction of watre uptake for the NaAlg/NaLS blend (80/20) in: a) $\mathrm{pH} 1.2$ and $\mathrm{pH} 7.4$; b) changing $\mathrm{pH}$; c) treated with $0.1 \mathrm{M} \mathrm{HCl}$ at different intervals in the medium of $\mathrm{pH} 7.4$. 
amorphous than pure samples of SA and LS, whereas among blends the SA/LS (60/40) appears to more amorphous than SA/LS(80/20). The increase in amorphous nature in blends leads to shrinkage in cell size or inter-segmental spacing occurring due to blending which would improve the selective permeation of the membrane.

\section{Fourier transform infrared spectroscopy (FT-IR) studies}

Fourier transform infrared (FT-IR) spectroscopy of blend films was carried out in order to detect any peak shift that could be attributed to weak interactions or structural changes between the two polymers. The FT-IR spectra of the interpolymer complexes show spectral features similar to those for the homopolymer ${ }^{[17]}$. Figure 4 shows the FT-IR spectra of the pure samples and blends in the wavelength ranges of 4000-2500 and 1600-1400 $\mathrm{cm}^{-1}$. The characteristic band of
LS was observed at $1600 \mathrm{~cm}^{-1}$ due to the aromatic structure. On the other hand, the bands of LS appeared at $3500 \mathrm{~cm}^{-1}$ for the hydroxyl groups. The spectrum of the SA/LS blend films was characterized by the presence of the absorption bands typical of the pure components, with the intensity roughly proportional to the blending ratio. The characteristic bands of SA appeared at 1611 and $3500 \mathrm{~cm}^{-1}$ were observed in all spectra of the blends. The spectra 1 (Figure $4 \mathrm{a}$ ) and 4 (Figure 4d) are attributed to single components SA and LS and they do not have much of noise, since the samples are in powder form. But the spectra 2 (Figure $4 \mathrm{~b}$ ) and 3 (Figure $4 \mathrm{c}$ ) possess more of noise and these are attributed to mixture of two components. Importantly, it can be observed that there is no appearance of new peaks when two components blended. This indicates that, blending might have taken place without any reaction between the two components leading to no structural change.

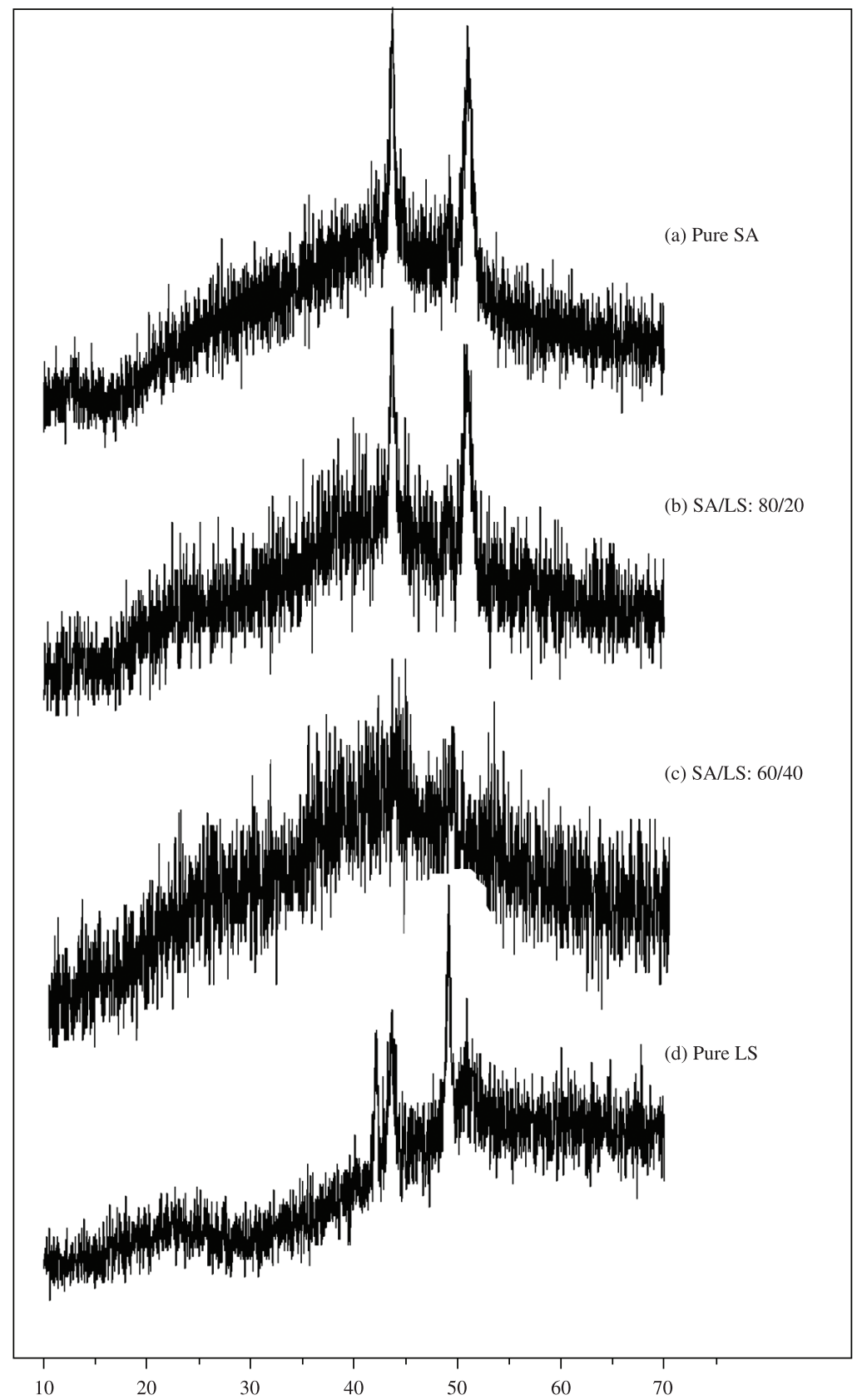

Figure 3. XRD of a) Pure SA; b) Blend 80/20; c) Blend 60/40; d) Pure LS. 
(a)

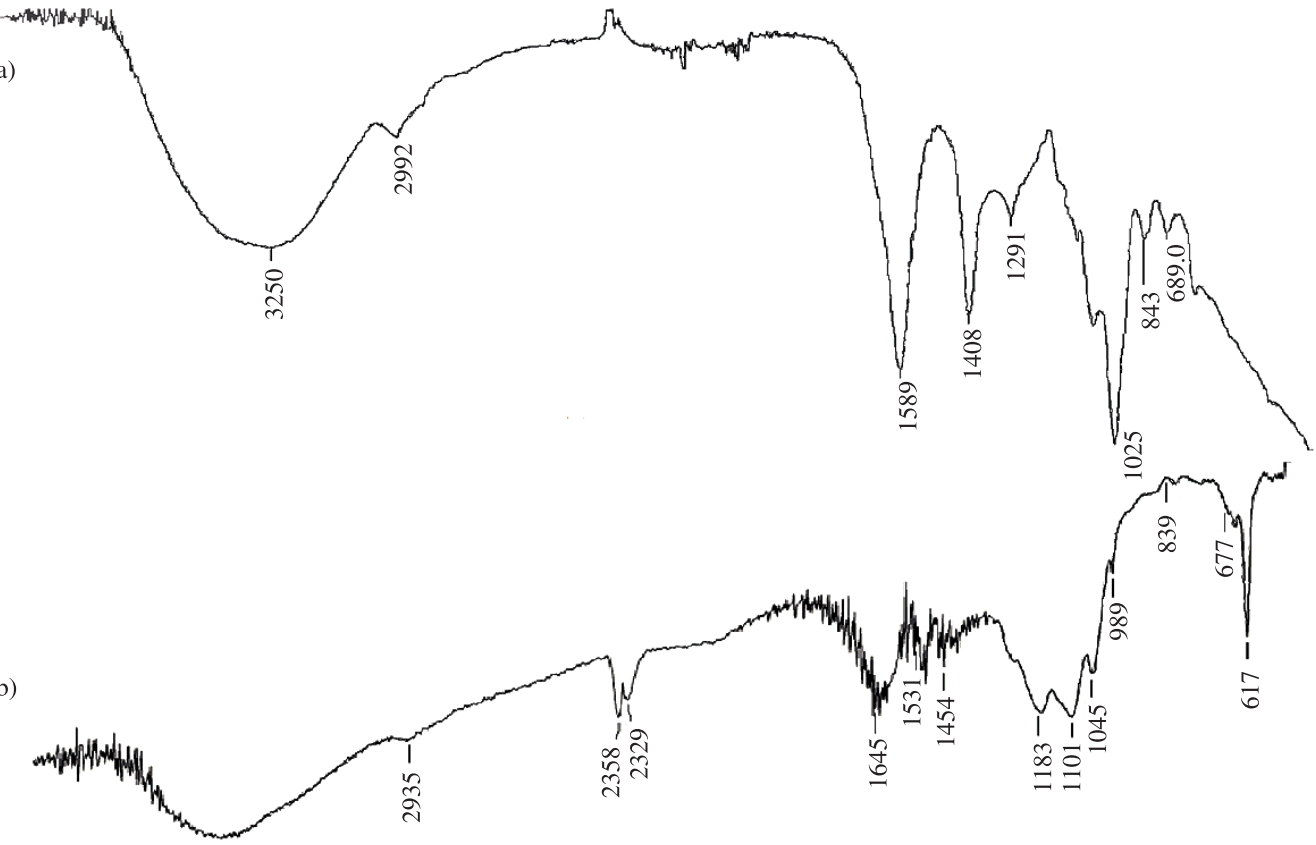

(c)
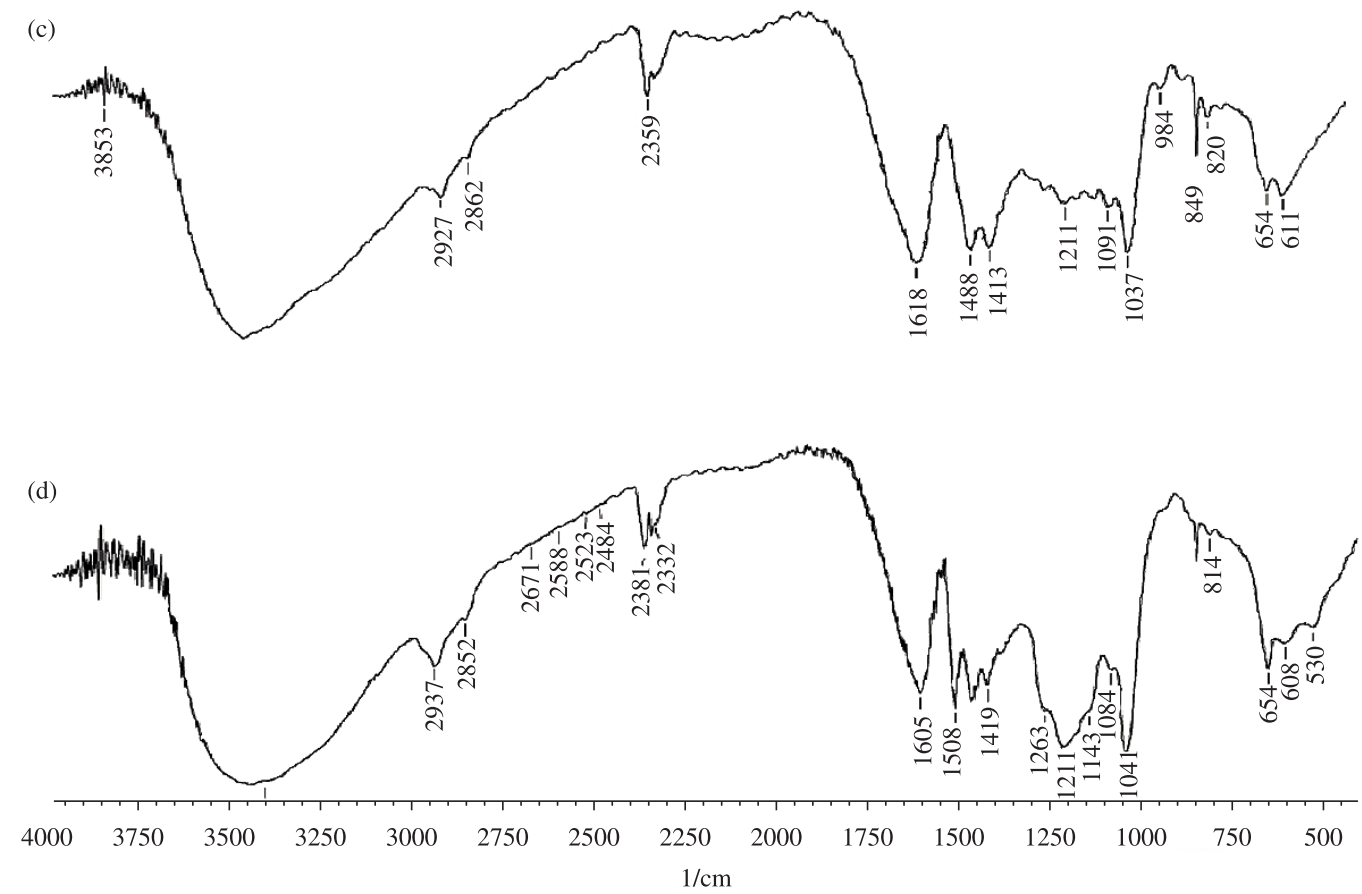

Figure 4. FTIR spectra of a) Pure NaAlg (100/0); b) NaAlg:NaLS (80/20); c) NaAlg: NaLS (60/40); d) Pure NaLS.

\section{Conclusion}

Blend films of LS and SA could be easily obtained by solution blending and casting onto the glass plate. The transport behavior of SA, SA/LS blends has been examined for water uptake in phosphate buffer of $\mathrm{pH}$ (7.4) as probe molecules at the room temperature. The strength of the blends and pure SA can be achieved by using calcium chloride as crosslinking agent for selective permeation. The solvent sorption decreases significantly with increase in LS content in SA. The decrease in solvent sorption, point towards the decrease in intermolecular spacing. The examination of influence of Calcium chloride at higher intervals shows more resistance to water uptake. The SA/LS blend (80/20) is subjected for the media of varying $\mathrm{pH}$ (1.2 \& 7.4), demonstrates $400 \pm 9 \%$ water uptake in 4 hours and demonstrates suitability to the possible usage for controlled drug delivery. Finally, swellings are conducted after acid treatment $(0.1 \mathrm{M}$ $\mathrm{HCl}$ ) and observed that swellings are altered drastically, due to decrosslinking. The XRD analysis confirms the decrease in crystalline regions in SA/LS blends. FT-IR analysis of SA and LS reveals that blending has taken place without any structural changes. 


\section{References}

1. Kim, S. W. \& Bae Okano, T. - Pharm. Res., 9, p.283 (1992). PMid:1614957. http://dx.doi.org/10.1023/A:1015887213431

2. Gutowska, A. \& Jeong Jasionowski, B. - Anat. Rec., 263, p.342 (2001). PMid:11500810. http://dx.doi.org/10.1002/ar.1115

3. Sakai, S.; Masuhara, H.; Yamada, Y.; Ono, T. \& Ijima, H. J. - Biosci. Bioeng., 100, p.127 (2005). PMid:16233864. http://dx.doi.org/10.1263/ jbb. 100.127

4. Zimmermann, H.; Zimmermann, D. \& Reuss, R. - J. Mater. Sci. Mater. Med., 16, p.491 (2005). PMid:15928863. http://dx.doi.org/10.1007/ s10856-005-0523-2

5. Smidsrod, O. \& Skjak-Braek, G. - Biotechnology, 8, p.71 (1990).

6. Lee, K. Y \& Mooney, D. J. - Chem. Rev., 101, p.1869 (2001). PMid:11710233. http://dx.doi.org/10.1021/cr000108x

7. Kwei, T. K.; Nishi, T. \& Roberts, R. F. - Macromolecules, 7, p.667 (1974). http://dx.doi.org/10.1021/ma60041a023

8. Johnson, T. \& Thomas, S. - Polym. Plast. Tech. Eng., 39, p.363 (2000). http://dx.doi.org/10.1081/PPT-100100035

9. Travinskaya, T. V. \& Savelyev, Y. V. - Eur. Polym. J., 42, p.388 (2006). http://dx.doi.org/10.1016/j.eurpolymj.2005.07.017
10. Sujith, A.; Unnikrishnan, G. \& Thomas, S. - J. Appl. Polym. Sci., 90, p.2691 (2003). http://dx.doi.org/10.1002/app.12923

11. Oh Jin, K.; Seung-Taek, O. H. \& Sang-Do, L. - Fibers Polym., 8, p.347 (2007).

12. Aslani, P. \& Kennedy, R. A. - J. Control. Release., 42, p.75 (1996). http://dx.doi.org/10.1016/0168-3659(96)01369-7

13. Subba Reddy, A.; Kalyani, S. \& Siva Kumar, N. - Polym. Bul., 61, p.779 (2008).

14. Krishnaiah, V. S. R.; Satyanarayan, S.; Rama Prasad, Y. V. \& Narsimharao, S. - J. Control. Release., 55, p.245 (1998). http://dx.doi. org/10.1016/S0168-3659(98)00057-1

15. Mumper, U. J.; Hoffman, A. S.; Puollakkainer, P. A.; Bouchard, L. S. \& Gombotz, W. R. - J. Control. Release., 30, p.241 (1994). http://dx.doi. org/10.1016/0168-3659(94)90030-2

16. Gombotz, W. R. \& Wee, S. F. - Adv. Drug Deliv. Rev., 31, p.267 (1998). http://dx.doi.org/10.1016/S0169-409X(97)00124-5

17. Tuncer Aykara, C.; Serkan Demircia, A. \& Mehmet, A. - Polymer, 46, p.10750 (2005)

Enviado: 06/03/12

Reenviado: 18/06/12

Aceito: $20 / 06 / 12$ 